\title{
Validación de la hipótesis de mortalidad por inanición en larvas de Engraulis ringens Jenyns, 1842 (Pisces: Engraulidae), utilizando el criterio histológico en un área de surgencia costera en el norte de Chile
}

\author{
Jessica Pizarro E., Winston Palma S. y Claudio Flores C. \\ Departamento Ciencias del Mar, Universidad Arturo Prat \\ Casilla 121, Iquique, Chile \\ E-mail: jessica.pizarro@cec.unap.cl
}

\begin{abstract}
RESUMEN. Se analiza la hipótesis de inanición como causa de la mortalidad natural en larvas de Engraulis ringens (anchoveta), en la época de otoño (abril) de 1989, en un área de surgencia costera del norte de Chile ( $20^{\circ} 30^{\prime} \mathrm{S}-21^{\circ} 45^{\prime}$ 'S). La condición nutricional de las larvas se determinó por criterios histológicos, examinando los tejidos del hígado, páncreas, intestino medio, musculatura, notocorda y cartílago. Se estudio un total de 6.226 larvas. La principal distribución espacial se presentó de 1 a $10 \mathrm{mn}$ de la costa y las mayores abundancias se registraron al sur del área de estudio. La longitud estándar de las larvas estudiadas fluctuaron entre 4,0 y 16,9, mm con un promedio de 8,5 mm. De los tres estados de condición nutricional observados, la condición saludable fue la que registró el mayor porcentaje $(57,1 \%)$, seguido de la semi-saludable $(40 \%)$ y de la de inanición $(2,9 \%)$. Se concluye que la mortalidad natural por inanición en esta especie sería poco relevante en la época y área de estudio.
\end{abstract}

Palabras claves: mortalidad, inanición, larvas, Engraulis ringens, caracterización histológica, Pacífico suroriental.

\author{
Validation of the hypothesis of mortality by starvation in \\ Engraulis ringens Jenyns, 1842 (Pisces: Engraulidae) larvae \\ using the histological criterion in an area of coast \\ upwelling in the north of Chile
}

\begin{abstract}
The hypothesis of starvation as a cause of natural mortality in larvae of Engraulis ringens (anchovy) in time autumm (april) 1989 is analyzed, in an area of coastal upwelling in the north of Chile (203' $\mathrm{S}-21^{\circ} 45^{\prime} \mathrm{S}$ ). The nutritional condition of the larvae was determined by histological criterion when tissues of liver, pancreas, midgut, musculature, notochord and cartilage were examined. A total of 6.226 larvae was studied. The main spatial distribution appeared between 1 to $10 \mathrm{mn}$ from the coast and the largest abundances were registered in the south of the area in study. The standard length of the larvae fluctuated between 4.0 and $16.9 \mathrm{~mm}$, with an average of $8.5 \mathrm{~mm}$. Out of the three states of nutritional condition observed, the healthful condition was the one that registred the greatest percentage of $(57.1 \%)$, followed by the semihealthful of $(40 \%)$ and the starvation $(2,9 \%)$. It is concluded that natural mortality by starvation of this specie would be of few relevance at this time and this in area of study.
\end{abstract}

Key words: mortality, inanition, larvae, Engraulis ringens, histological criterion, Southeast Pacific.

\section{INTRODUCCION}

Las especies de peces pelágicos, particularmente los Clupeiformes, experimentan grandes fluctuaciones en las abundancias de sus poblaciones motivadas, particularmente, por la alta variabilidad del reclutamiento (Blaxter y Hempel, 1963; Santander y Flo- res, 1983). Este aspecto ha motivado que se intensifique una serie de investigaciones referente al estudio de los mecanismos o causas que afectan la variación de este proceso, conocimiento muy importante en dinámica de poblaciones. Entre las hipóte- 
sis que se manejan para dilucidar el tamaño de una nueva clase anual está la propuesta por Hjort (1914), quien sugirió que el éxito en el reclutamiento estaba determinado en los primeros estados de la vida de los peces, en particular en su estado de larva, que en general son mucho más sensibles a la calidad del ambiente. La disponibilidad de alimento a la edad de primera alimentación, es una de las causas relevantes de mortalidad. En este período, el éxito de la alimentación y consecuentemente su crecimiento individual y supervivencia, en que la larva pasa de una alimentación endógena (vitelínica) a una exógena, dependerá de la concentración, tamaño, tipo y valor nutritivo de las partículas, como también del comportamiento y estrategias del depredador como de la presa, capacidad de locomoción, percepción sensorial, éxito de la captura y escape, entre otros (Lasker y Zweifel, 1978).

En la actualidad hay mayores evidencias para poner a prueba esta hipótesis, existiendo numerosos estudios de laboratorio que han demostrado que la mortalidad por desnutrición de las larvas de peces es una causa de mortalidad natural, que puede ser detectada utilizando principalmente los métodos morfométricos e histológicos (Umeda y Ochiai, 1975; O’Connell, 1976; Ehrlich et al., 1976; Theilacker, 1978; Govoni, 1980; Martin y Malloy, 1980; Martin y Wright, 1987; Theilacker y Watanabe, 1989), así como también por criterios bioquímicos basados en la relación ARN/ADN (Buckey, 1979, 1984; Wright y Martin 1985; Clemmense, 1987, 1988). Los estudios cuantitativos de mortalidad por inanición en el medio marino son escasos en comparación con las experiencias de laboratorio, destacando los trabajos de O'Connell (1981) en larvas de Engraulis mordax de la costa sur de California; Theilacker (1986) en Trachurus symmetricus frente a la costa de California y para la costa chilena, los realizados en Sardinops sagax frente a Quintero y Valparaíso (Uriarte y Balbontín, 1987), y en Engraulis ringens frente a Talcahuano (Herrera com. pers.).

Frente a la desembocadura del río Loa en el norte de Chile, entre los $20^{\circ} 30^{\prime} \mathrm{S}-21^{\circ} 45^{\prime} \mathrm{S}$, se han encontrado históricamente concentraciones importantes de huevos y larvas de Engraulis ringens (Palma et al., 1992). Las condiciones físicas de esta área oceánica en la época de estudio se caracterizan principalmente por índices positivos de surgencia (Fuenzalida, 1992), patrón permanente y normal, que se manifiesta en una producción biológica promedio de biomasa integrada de fitoplancton y zooplancton de $12,7 \mathrm{mg}$ de clorofila $\mathrm{a} / \mathrm{m}^{3}$ y 2.370 $\mathrm{ml}$ de zooplancton $/ 1.000 \mathrm{~m}^{3}$ respectivamente. Si se considera que el alimento es uno de los factores fundamentales para la sobrevivencia de las larvas y que determinaría el éxito de una nueva clase de edad, entonces, para la zona del río Loa, se esperaría que la hipótesis de inanición como causa de la mortalidad larval no fuera relevante. En el presente estudio se pretende poner a prueba esta hipótesis en larvas de Engraulis ringens en un área de alta producción biológica, determinando cuantitativamente la condición nutricional de las larvas en relación a su distribución espacial, abundancia y estructura de talla.

\section{MATERIALES Y METODOS}

Las muestras ictioplanctónicas fueron obtenidas en abril de 1989, a bordo del B/I Carlos Porter, en la zona norte de Chile frente a la costa del río Loa entre Caleta Chucumata $\left(20^{\circ} 30^{\prime} \mathrm{S}\right)$ y Punta Urcus $\left(21^{\circ} 45^{\prime} \mathrm{S}\right)$. El diseño de muestreo consistió en seis transectas perpendiculares a la costa separadas por $15 \mathrm{mn}$, cada una con cuatro estaciones distribuidas a $1,5,10$ y $30 \mathrm{mn}$, cubriendo una superficie total de $2.383 \mathrm{mn}^{2}$ (Fig. 1). La recolección de las muestras se realizó mediante arrastres oblicuos entre 0 y 200 metros de profundidad con una red bongo de $300 \mu \mathrm{m}$ y $500 \mu \mathrm{m}$ de malla y $0,273 \mathrm{~m}^{2}$ de abertura de boca a una velocidad de 1,5 nudos. En este estudio sólo se analizaron las larvas obtenidas con la red de $500 \mu \mathrm{m}$. Las muestras fueron preservadas inmediatamente en solución Bouin, procurando que no pasara más de un minuto entre la muestra en cubierta y su fijación, manteniéndose así por dos horas y luego fueron transferidas a alcohol $70^{\circ}$. En el laboratorio las larvas de anchoveta se separaron del resto del zooplancton con un microscopio estereoscópico; luego fueron contadas y medidas su longitud estándar (LE) con un lente micrométrico de $100 \mu \mathrm{m}$ de precisión.

Las larvas se sometieron a la técnica histológica, que consistió en deshidratarlas a través de una batería de alcoholes entre 70 y 100\%, finalizando con butanol. Luego se impregnaron e incluyeron in toto en paraplast. Luego se realizaron cortes en forma seriada en un plano sagital con un espesor de $5 \mu \mathrm{m}$; éstos fueron teñidos con hematoxilina y eosinaphloxine $B$ y montados en entellan (Theilacker, 1978).

Para la evaluación de la condición nutricional se examinó microscópicamente la histoarquitectura de los tejidos cartilaginoso, muscular del tronco, 


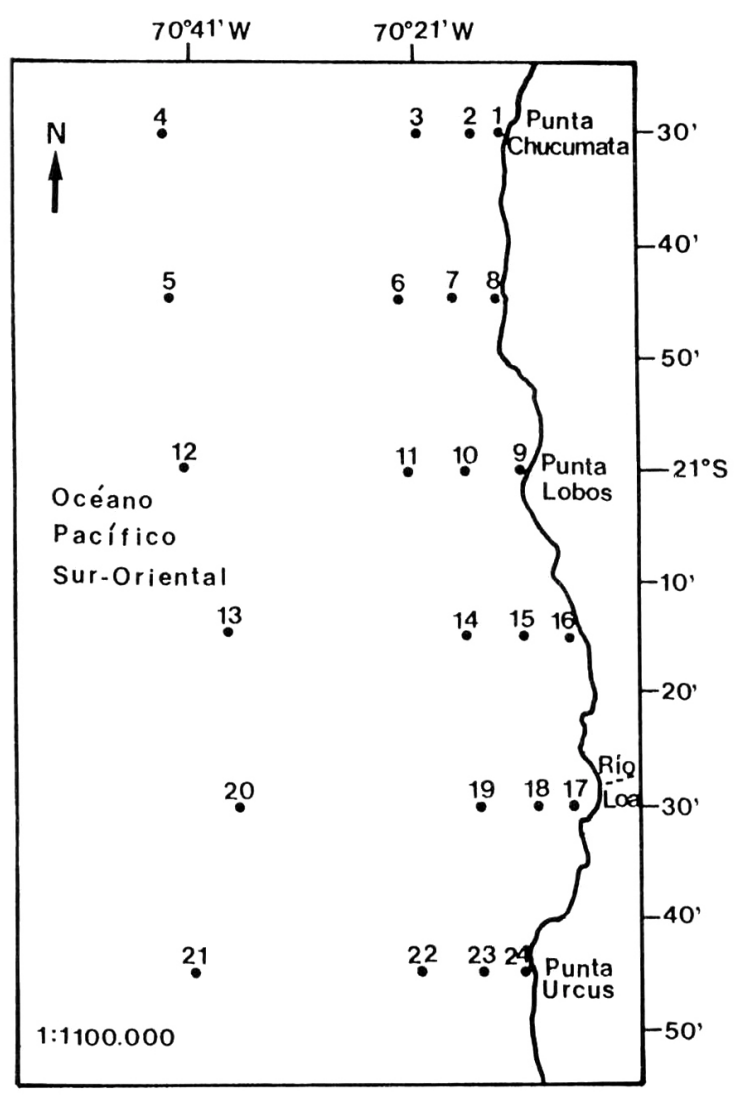

Figura 1. Area de estudio y ubicación geográfica de las estaciones de muestreo.

Figure 1. Area of study and geographycal location of the sampling stations.

intestino medio, notocorda, páncreas e hígado. Para lo cual se siguió el criterio propuesto por O'Connell (1976), donde cada uno de los tejidos se evalúa asignándole un puntaje de 1 a 3 de acuerdo a las características nutricionales; así, un nivel 3 correspondió a tejido saludable o normal (larvas alimentadas), 2 a semisaludable o intermedias (con temprana desnutrición) y 1 a inanición (desnutridas o moribundas). La decisión para definir la condición nutricional de cada larva correspondió al promedio de la evaluación de todos los tejidos: 2,34 a 3,0 para la condición saludable; 1,67 a 2,33 para la semisaludable y 1,0 a 1,66 para la inanición.

Para estimar la abundancia de larvas por estado de condición nutricional $\left(\mathrm{N}^{\mathrm{o}}\right.$ larvas $\left./ 10 \mathrm{~m}^{2}\right)$, se utilizó la ecuación de estandarización $\mathrm{C}=10 *\left(\mathrm{a}^{-1} * \mathrm{~b}^{-1}\right.$ $* \mathrm{c} * \mathrm{~d}$ ), donde: (C) es el número de larvas por unidad de área de mar $\left(10 \mathrm{~m}^{2}\right)$; (a) el área de la boca de la red bongo en $\mathrm{m}^{2}$; (b) el largo de la trayectoria del arrastre en metros; (c) el número de larvas en la muestra y (d) la profundidad máxima del lance en metros. Para estimar el total de larvas por estado de condición nutricional, se utilizó el índice larval L $=\mathrm{F} *\left(\mathrm{P} * \mathrm{C}^{\prime}\right)$, donde: $(\mathrm{L})$ es el índice larval; $(\mathrm{F})$ el factor areal; (P) la proporción de estaciones positivas y $\left(C^{`}\right)$ el número promedio de larvas por estaciones positivas (Smith y Richardson, 1979).

Para el análisis de distribución de abundancia se utilizó la escala geométrica de Frontier (1966) y para la distribución de los estados de condición nutricional se estratificó el área utilizando como criterio la distancia en millas desde de la costa, obteniendo cuatro estratos (Estrato I: distancia a $1 \mathrm{mn}$ de la costa (Estación: 1, 8, 9, 16, 17, 24), Estrato II: distancia a $5 \mathrm{mn}$ de la costa (Estación: 2, 7, 10, 15, 18, 23), Estrato III: distancia a $10 \mathrm{mn}$ de la costa (Estación: 3, 6, 11, 14, 19, 22) y Estrato IV: distancia a $30 \mathrm{mn}$ de la costa (Estación: 4, 5, 12, 13, 20, 21). La comparación de abundancia de los diferentes estados de condición por estrato se realizó mediante una ANOVA de dos vías (Sokal y Rohlf, 1969).

\section{RESULTADOS}

\section{Caracterización histológica de las larvas por estado de condición nutricional}

El tejido cartilaginoso de las larvas de anchoveta en condición saludable se caracteriza por poseer condriocitos con núcleo redondeado y espacio capsular completo de citoplasma; en condición semisaludable el núcleo presenta grados variables de condensación y el citoplasma no ocupa completamente el espacio capsular; y en condición de inanición el núcleo está condensado y el citoplasma muy restringido o ausente (Figs. 2A a 2C).

La musculatura en condición saludable se caracteriza principalmente porque el tejido es compacto y uniforme sin espacios intermusculares distinguibles, en el núcleo se observa claramente el nucléolo; en semisaludable se distinguen separaciones leves entre las fibras musculares; y en inanición se observan amplios espacios intermusculares con una disposición desordenada de las fibras y los núcleos están esparcidos irregularmente (Figs. 2D a 2F).

El intestino en condición saludable se caracteriza porque las células de la pared son compactas, sin separación entre ellas y el citoplasma es homogéneo, el lumen intestinal es amplio; en semisaludable 

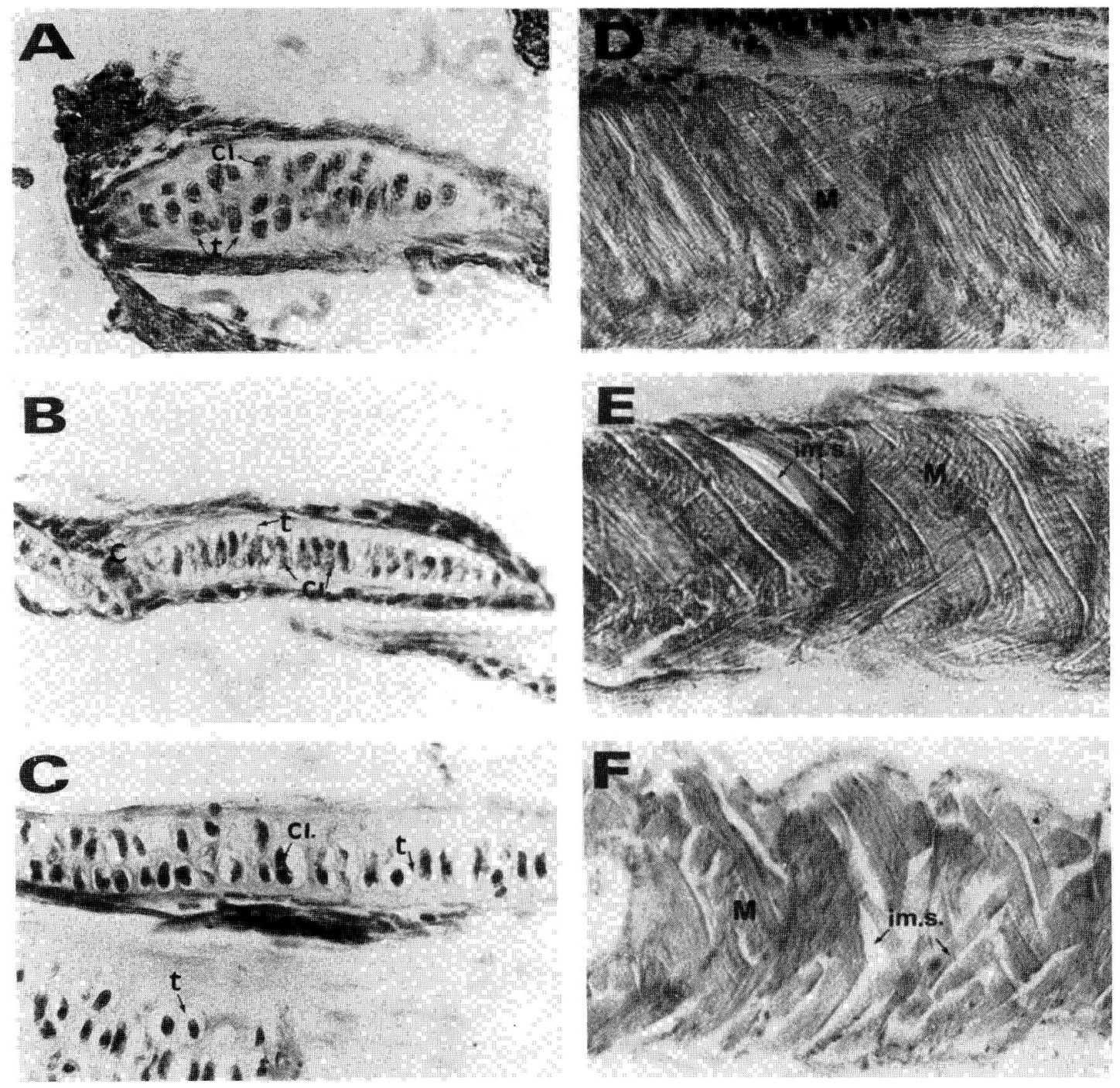

Figura 2. Caracterización histológica del cartílago y musculatura de larvas de Engraulis ringens. A) cartílago saludable (40x); B) cartílago semi-saludable (40x); C) cartílago en inanición (40x); D) musculatura saludable (40x); E) musculatura semi-saludable (40x); F) musculatura en inanición (40x). Simbología: (CI) citoplasma, (t) cápsula, (M) musculatura, (IM.S) espacios intermusculares.

Figure 2. Histological characterization of the cartilage and musculature of Engraulis ringens larvae. A) healthy cartilage (40x); B) semi-healthy cartilage $(40 x)$; C) in starvation cartilage (40x); D) healthy musculature (40x); E) semi-healthy musculature $(40 x) ; F)$ in starvation musculature $(40 x)$. Symbology: (CI) cytoplasm, (t) capsule, (M) musculature, (IM.S) intermuscular space. 
se observan separaciones leves entre las células; y en inanición las células están separadas y el citoplasma es oscuro (Figs. 3A a 3D).

La notocorda en condición saludable se caracteriza por no presentar separación entre ella y la musculatura; en semisaludable se distinguen zonas de separación con la musculatura; y en inanición la notocorda se encuentra contraída y ampliamente separada con la musculatura (Figs. 3E y 3F).

El páncreas en condición nutricional saludable presenta el tejido compacto, el nucléolo es observable en el núcleo y el ducto pancreático está completo de cimógeno; en semisaludable el tejido presenta cierto grado de disociación con algunos espacios intercelulares; y en inanición el tejido está fragmentado, las células son pequeñas, separadas y de forma irregular (Figs. 4A a 4D).

El hígado en condición saludable presenta el tejido compacto, núcleos grandes y nucléolos distinguibles, citoplasma con espacios intracelulares; en semisaludable el tejido presenta cierto grado de disociación, el citoplasma con menor vacuolización (espacios intracelulares); y en inanición el tejido está fragmentado, citoplasma reducido y sin espacios intracelulares, núcleos intensamente teñidos y nucléolos no distinguibles (Figs. 4A a 4F).

\section{Abundancia larval por estados de condición nutricional}

Se analizó un total de 6.226 larvas, de las cuales 3.550 fueron clasificadas en condición nutricional saludable, 2.498 en semisaludable y 178 en inanición. Las larvas en inanición presentaron su mayor abundancia en la estación 24 con 62 larvas $/ 10 \mathrm{~m}^{2}$ y la menor en la estación 10 con 11 larvas $/ 10 \mathrm{~m}^{2}$, lo que representa un $13,6 \%$ y $2,0 \%$ para cada estación respectivamente. Asimismo, las larvas semisaludables presentaron la mayor abundancia en la estación 17 con 700 larvas/10 m² y la menor en la estación 5 con 14 larvas $/ 10 \mathrm{~m}^{2}$, que representan un $47,9 \%$ y $100 \%$ respectivamente. Para la condición saludable, la estación mejor representada correspondió a la 17 con 740 larvas/10 $\mathrm{m}^{2}$ y la de menor abundancia a la estación 8 con 12 larvas $/ 10 \mathrm{~m}^{2}$, que representa el $50,7 \%$ y $100 \%$ respectivamente (Tabla $1)$.

\section{Distribución espacial}

Las larvas en condición nutricional saludable se distribuyeron desde los $20^{\circ} 30^{\prime} \mathrm{S}$ a $\operatorname{los} 21^{\circ} 45^{\prime} \mathrm{S}$, las semisaludables desde $20^{\circ} 45^{\prime} \mathrm{S}$ a $21^{\circ} 45^{\prime} \mathrm{S}$ y en inani- ción sólo se registraron en cinco estaciones ubicadas en la franja costera a $1 \mathrm{mn}$ (Estaciones 16, 17, 24) y a $5 \mathrm{mn}$ (Estaciones 10 y 23), entre los $21^{\circ} \mathrm{S}$ y $21^{\circ} 45^{\prime} \mathrm{S}$. En general, las larvas se encontraron entre 1 a $10 \mathrm{mn}$ y en una sola estación a $30 \mathrm{mn}$ (Estación 5), donde todas presentaron una condición semisaludable.

Asimismo, se detectaron concentraciones de larvas donde dominaba un estado de condición y en otras una combinación de los tres estados nutricionales. Así, por ejemplo, se observaron sólo larvas saludables en las estaciones 1,2 y 8 entre 1 y $5 \mathrm{mn}$ de la costa frente a Punta Chucumata, y en la estación 18 ubicada a $5 \mathrm{mn}$ de la desembocadura del río Loa, y solamente semisaludables en la estación 22 a $10 \mathrm{mn}$ frente a Punta Urcus y en la estación 5 a 30 mn de la costa.

Tabla 1. Abundancia de larvas de Engraulis ringens (larvas/10 $\mathrm{m}^{2}$ ) por estado de condición nutricional.

Table 1. Abundance of Engraulis ringens larvae (larvae/10 $\mathrm{m}^{2}$ ) by state of nutritional condition.

\begin{tabular}{|rrrrr|}
\hline Estación & Inanición & $\begin{array}{c}\text { Semi- } \\
\text { saludable }\end{array}$ & Saludable & Total \\
\hline 01 & 0 & 0 & 36 & 36 \\
02 & 0 & 0 & 109 & 109 \\
03 & 0 & 0 & 0 & 0 \\
04 & 0 & 0 & 0 & 0 \\
05 & 0 & 14 & 0 & 14 \\
06 & 0 & 0 & 0 & 0 \\
07 & 0 & 24 & 47 & 71 \\
08 & 0 & 0 & 12 & 12 \\
09 & 0 & 77 & 145 & 222 \\
10 & 11 & 218 & 317 & 546 \\
11 & 0 & 50 & 75 & 125 \\
12 & 0 & 0 & 0 & 0 \\
13 & 0 & 0 & 0 & 0 \\
14 & 0 & 264 & 422 & 686 \\
15 & 0 & 76 & 171 & 247 \\
16 & 33 & 167 & 284 & 484 \\
17 & 20 & 700 & 740 & 1.460 \\
18 & 0 & 0 & 183 & 183 \\
19 & 0 & 174 & 290 & 464 \\
20 & 0 & 0 & 0 & 0 \\
21 & 0 & 0 & 0 & 0 \\
22 & 0 & 41 & 0 & 41 \\
23 & 52 & 548 & 470 & 1.070 \\
24 & 62 & 145 & 249 & 456 \\
\hline$\ldots$ Total & 178 & 2.498 & 3.550 & 6.226 \\
\hline
\end{tabular}



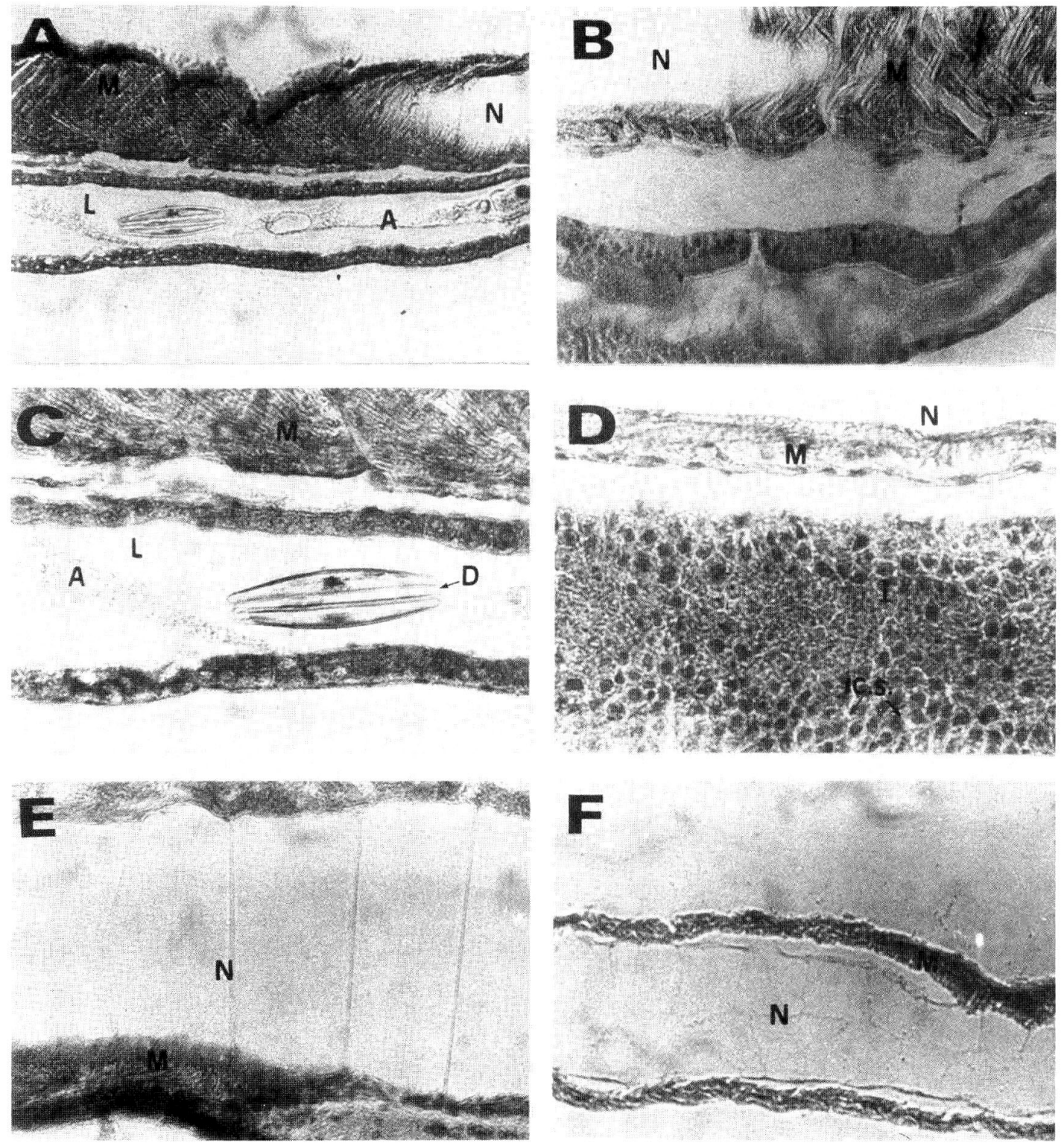

Figura 3. Caracterización histológica del intestino y notocorda de larvas de Engraulis ringens. A y C) intestino saludable (A:20x, C:40x); B y D) intestino en inanición (B:20x, D:40x); E) notocorda saludable (40x); F) notocorda en inanición (20x). Simbología: (N) notocorda, (L) lumen, (A) alimento, (I) intestino, (D) diatomea del género Navicula, (IC.S) espacios intercelulares.

Figure 3. Histological characterization of the gut and notochord of Engraulis ringens larvae. A y C) healthy gut $(A: 20 x, C: 40 x) ; B y ~ D)$ in starvation gut (B:20x, D:40x); E) healthy notochord (40x); F) in starvation notochord (20x). Symbology: (N) notochord, (L) lumen, (A) food, (I) gut, (D) diatoms of genus Navicula, (IC.S) intercellular space. 

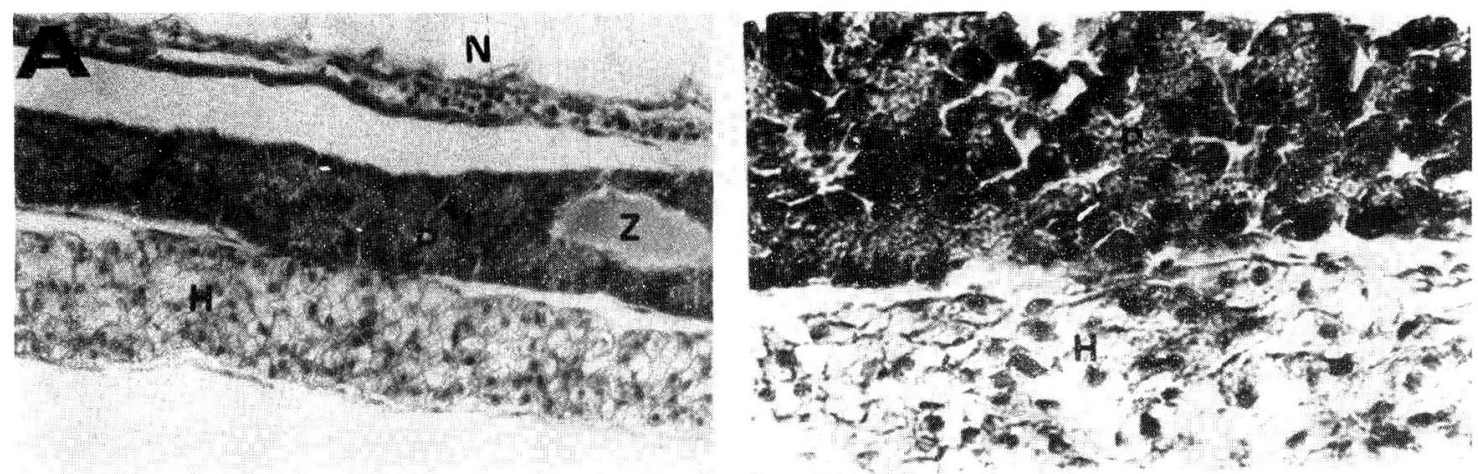
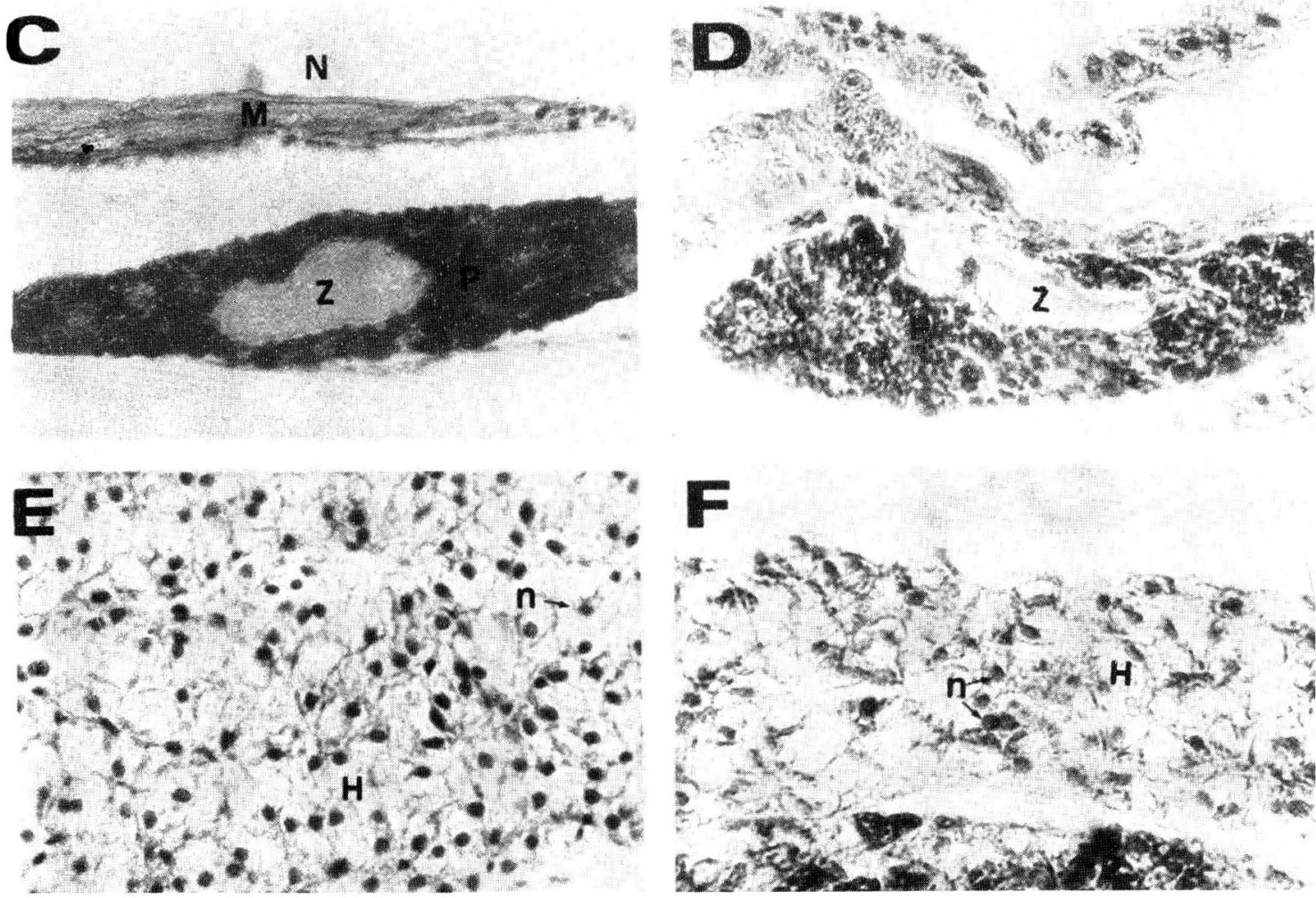

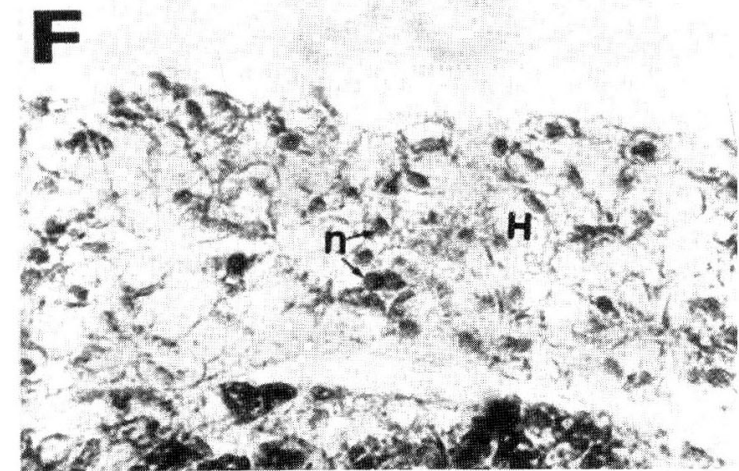

Figura 4. Caracterización histológica del páncreas e hígado de larvas de Engraulis ringens. A) páncreas e hígado saludables (20x); B) páncreas e hígado en inanición (40x); C) páncreas saludable (40x); D) páncreas en inanición (40x); E) hígado saludable (40x); F) hígado en inanición (40x). Simbología: (P) páncreas, (H) hígado, (Z) cimógeno, (n) núcleo, $(\mathrm{M})$ musculatura, $(\mathrm{N})$ notocorda.

Figure 4. Histological characterization of the pancreas and liver of Engraulis ringens larvae. A) healthy pancreas and liver $(20 x) ; B)$ in starvation pancreas and liver $(40 x) ; C)$ healthy pancreas $(40 x)$; D) in starvation pancreas $(40 x)$; E) healthy liver $(40 x)$; F) in starvation liver (40x). Symbology: (P) pancreas, (H) liver, $(Z)$ zimogen, n) nuclei, (M) musculature, (N) notochord. 


\section{Estructura de talla}

Los rangos de tallas fluctuaron entre 4,0 y $16,9 \mathrm{~mm}$ de LE, observándose una mayor frecuencia en el rango de $8,0-8,9 \mathrm{~mm}$ con un $24,4 \%$, seguida del tamaño $9,0-9,9 \mathrm{~mm}$ con $17,6 \%$. Las menores frecuencias se observaron en el rango de 13,0 a $16,9 \mathrm{~mm}$ con frecuencias entre 0,34 y $1,19 \%$.

La estructura de talla según su estado de condición, indica que las larvas saludables presentan una gran variabilidad entre 4,0 y $16,9 \mathrm{~mm}$, siendo las tallas entre 8,0 y $9,9 \mathrm{~mm}$ las más frecuentes. En las larvas semisaludables los rangos de talla varían entre 4,0 y $12,9 \mathrm{~mm}$ siendo las más frecuentes entre los 8,0-8,9 mm, mientras que para las larvas en inanición existen dos grupos de tallas, uno de 5,0-5,9 $\mathrm{mm}$ y otro de 8,0-11,9 mm, con frecuencias entre 0,32 y $1,01 \%$ (Fig. 6).

\section{Condición nutricional de larvas por estaciones positivas}

De un total de 24 estaciones muestreadas, 17 fueron positivas con larvas de anchoveta, lo que correspondió a una proporción de 0,71 . El prostudy. medio de larvas por estación positiva fue de 366 larvas (Tabla 2).

Con relación a los estados de condición nutricional, las larvas saludables se encontraron en 15 estaciones positivas con una proporción de 0,62 , las larvas semisaludables en 13 estaciones con una proporción de 0,54 y las larvas en inanición en 5 estaciones positivas, con una proporción de 0,20. El promedio de larvas saludables por estación positiva fue de 237, semisaludables 192 y en inanición 36 (Tabla 3).

Tabla 2. Estaciones positivas con larvas de E. ringens en el área de estudio.

Table 2. Positive stations with larvae of $E$. ringens at the area of

\begin{tabular}{|lcccc|}
\hline Especie & $\begin{array}{c}\text { Número de } \\
\text { estaciones } \\
\text { ocupadas }\end{array}$ & $\begin{array}{c}\text { Número de Proporción de Promedio larvas } \\
\text { estaciones } \\
\text { con larvas }\end{array}$ & $\begin{array}{c}\text { estaciones } \\
\text { positivas } \\
\text { (P) }\end{array}$ & $\begin{array}{c}\text { por estación } \\
\text { positiva } \\
\text { (C`) }\end{array}$ \\
\hline E. ringens & 24 & 17 & 0,71 & 366 \\
\hline
\end{tabular}

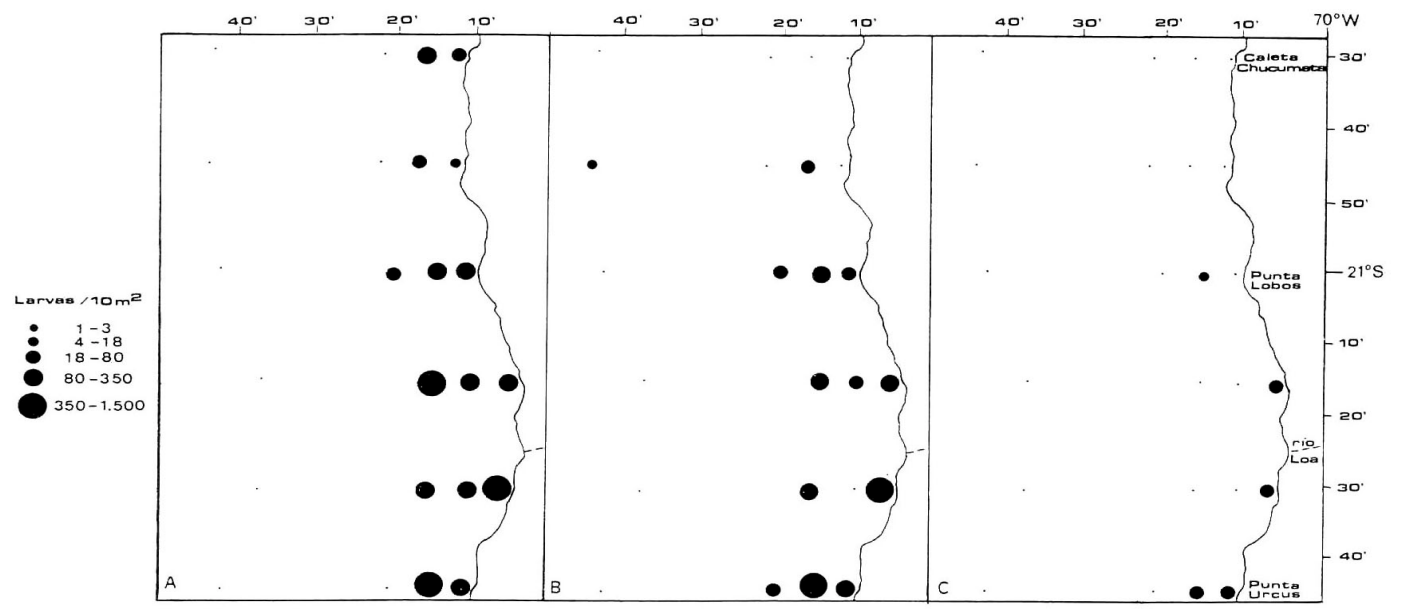

Figura 5. Distribución espacial de larvas de Engraulis ringens por estado de condición nutricional. a) larvas saludables, b) larvas semi-saludables, c) larvas en inanición.

Figure 5. Spatial distribution of Engraulis ringens larvae by state of nutritional condition. a) healthy larvae, b) semi-healthy larvae, c) in starvation larvae. 
Tabla 3. Estaciones positivas con larvas de $E$. ringens por estado de condición nutricional.

Table 3. Positive stations with larvae of E. ringens by state of nutritional condition.

\begin{tabular}{|lcccc|}
\hline $\begin{array}{l}\text { Estado de } \\
\text { condición } \\
\text { nutricional }\end{array}$ & $\begin{array}{c}\text { Número de } \\
\text { estaciones } \\
\text { ocupadas }\end{array}$ & $\begin{array}{c}\text { Número de } \\
\text { estaciones } \\
\text { con larvas }\end{array}$ & $\begin{array}{c}\text { Proporción de } \\
\text { estaciones } \\
\text { positivas }\end{array}$ & $\begin{array}{c}\text { Promedio } \\
\text { lar vas } \\
\text { por estación } \\
\text { positiva } \\
\left(\mathbf{C}^{6}\right)\end{array}$ \\
\hline$\ldots$ & & & $(\mathbf{P})$ & 237 \\
$\begin{array}{l}\text { Saludable } \\
\text { Semi-saludable }\end{array}$ & 24 & 15 & 0,62 & 192 \\
Inanición & 24 & 13 & 0,54 & 36 \\
\hline
\end{tabular}

Tabla 4. Indice larval calculado para $E$. ringens en el área de estudio por estados de condición nutricional (área total: 2.383 $\mathrm{m}^{2}$; factor areal: $\left.8,7 * 10^{8}\left(10 \mathrm{~m}^{2}\right)\right)$.

Table 4. Larval index calculated for $E$. ringens in the area of study by nutritional condition states (total area: $2.383 \mathbf{~ m n}^{2}$; areal factor: $\left.8,7 * 10^{8}\left(10 \mathrm{~m}^{2}\right)\right)$.

\begin{tabular}{|lcccc|}
\hline $\begin{array}{l}\text { Estado de Indice larval Porcentaje } \\
\text { condición } \\
\text { Nutricional }\end{array} \quad \mathbf{( L )}$ & $\mathbf{( \% )}$ & $\begin{array}{c}\text { Intervalo de Confianza } \\
(\mathbf{9 5} \%)\end{array}$ \\
\hline $\begin{array}{l}\text { Saludable } \\
\text { Semi- } \\
\text { saludable }\end{array}$ & $1,21 * 10^{11}$ & 57,1 & $5,70 * 10^{10}$ & $£ 1,21 * 10^{11} £ 1,21 * 10^{11}$ \\
Inanición & $6,11 * 10^{10}$ & 40,0 & $1,21 * 10^{11} £ 1,21 * 10^{11} £ 1,21 * 10^{11}$ \\
\hline Total & $2,11 * 10^{11}$ & 100 & $0 £ 6,11 * 10^{9} £ 2,45 * 10^{10}$ \\
\hline
\end{tabular}

Tabla 5. Anova de la abundancia de larvas de $E$. ringens por estado de condición nutricional entre diferentes distancias de la costa (estratos I, II, III, IV). (* = diferencia significativa al 95\%, $\mathrm{ns}=$ diferencia no significativa).

Table 5. Anova of the abundance of $E$. ringens larvae by state of nutritional condition between different distances from the coast (layers I, II, III, IV). (* = significant differences $95 \%, \mathrm{~ns}=$ nonsignificant differences).

\begin{tabular}{|lcccc|}
\hline $\begin{array}{l}\text { Fuente de } \\
\text { Variación } \\
\text { (gl) }\end{array}$ & $\begin{array}{c}\text { Grados de } \\
\text { Libertad } \\
\text { (S.C.) }\end{array}$ & $\begin{array}{c}\text { Suma de } \\
\text { Cuadrados } \\
\text { (M.S.) }\end{array}$ & $\begin{array}{c}\text { Cuadrado } \\
\text { Medio }\end{array}$ & F \\
\hline $\begin{array}{l}\text { Entre estados } \\
\text { de condición }\end{array}$ & 2 & 14,69 & 7,34 & $11,36^{*}$ \\
$\begin{array}{l}\text { Entre estratos } \\
\text { (I, II, II, IV) }\end{array}$ & 11 & 22,14 & 7,38 & $11,41^{*}$ \\
Interacción & 6 & 6,37 & 1,06 & $1,64 \mathrm{~ns}$ \\
\hline
\end{tabular}

Estimación del índice larval por estado de condición nutricional

El índice larval correspondió a $2,11^{*} 10^{11}$ en el área total de $2.383 \mathrm{mn}^{2}$. A las larvas en condición nutricional saludable correspondió a $1,21 * 10^{11}$, con un porcentaje de $57,1 \%$; a las semisaludables $8,48 * 10^{10}$, con un $40 \%$; y para las en inanición $6,11 * 10^{9}$, con un $2,9 \%$. El estado de inanición fue inferior en un orden de magnitud, con respecto al semisaludable y dos órdenes en relación al saludable (Tabla 4).

\section{Análisis de varianza de los estados de condición nutricional por estratos}

Del análisis de varianza se comprueba que existen diferencias significativas $(p<0,05)$ entre las diferentes distancias de la costa (estratos I, II, III, IV), como también entre los estados de condición (saludable, semisaludable, inanición), no existiendo diferencias significativas ( $p>0,05)$ en el análisis de interacción entre los estados de condición por estratos (Tabla 5).

\section{DISCUSION}

El porcentaje de larvas en condición de inanición (2,9\%) es comparativamente menor a lo informado en otros estudios. Por ejemplo, O'Connell (1981) encontró larvas de anchoveta del Pacífico norte (Engraulis mordax) con una mortalidad diaria por inanición de $8 \%$ en el rango de talla de 2,5-10,0 mm (LE). Theilacker (1986) en larvas de Trachurus symmetricus detectó un $57-70 \%$ de larvas en inanición colectadas mar afuera en la zona de California, en contraste a un 6-12\% de larvas capturadas cerca de la costa. Uriarte y Balbontín (1987) detectaron un $16 \%$ de larvas de sardina Sardinops sagax con síntomas de inanición en aguas costeras frente a Chile central. En este estudio, las larvas más pequeñas encontradas en estado de inanición estuvieron alrededor de los $5,5 \mathrm{~mm}$ (LE). Cabe destacar que registros de lar- 


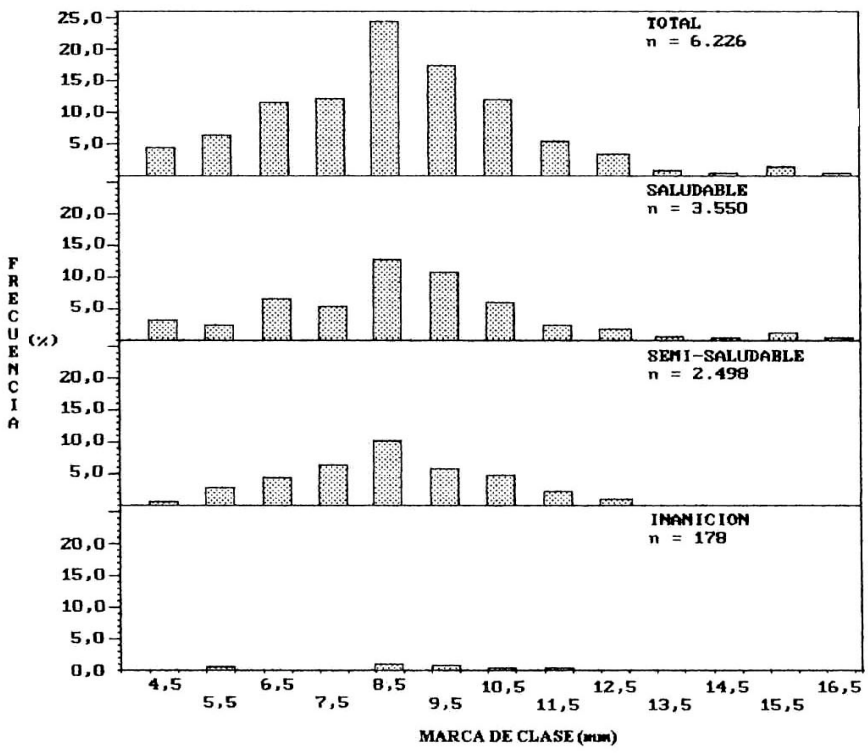

Figura 6. Distribución de frecuencia de talla $(\mathrm{mm})$ de larvas de Engraulis ringens por estados de condición nutricional.

Figure 6. Size frecuency distribution $(\mathrm{mm})$ of Engraulis ringens larvae by states of nutritional condition.

vas de anchoveta con alimentación endógena (estado de saco vitelino), encontradas en la zona de estudio, presentaron tallas alrededor de una media de $3,7 \mathrm{~mm}$ (LE) y las larvas más pequeñas de primera alimentación (boca funcional) un tamaño de 4,0 mm (LE). Las tallas de primera alimentación varían de acuerdo a la especie y al hábitat donde se desarrollan los primeros estados de vida. De este modo, las comparaciones de los porcentajes de larvas en inanición con otros estudios, se efectuaron considerando sólo la condición fisiológica de larvas con boca funcional.

O'Connell y Raymond (1970) y Hunter (1972) en experimentos de laboratorio han demostrado que las larvas pueden requerir altas concentraciones de microplancton para no morir de inanición durante las etapas iniciales (primera semana posterior al estado de saco vitelino). Sin embargo, Blaxter y Ehrlich (1974) demuestran que es imposible que las larvas no tengan alimento en el mar. La respuesta del organismo es en este caso, cuando falta el alimento, es la de prolongar su estado de supervivencia. Por otra parte, es improbable que en zonas de surgencias se encuentren bajas concentraciones de alimento para no poder satisfacer las necesidades metabólicas de las larvas. En consecuencia, se postula que éste sería el motivo de los bajos porcentajes de larvas en inanición encontrados en el área de estudio, la cual es altamente productiva debido a procesos permanentes de surgencia costera (Fuenzalida, 1990). Owen (1980, 1981a, 1981b), estudiando 1a microdistribución de parches de alimento en áreas de surgencia, como las costas de California y frente al Perú, encontró que éstos son adecuados para la sobrevivencia de larvas.

Por otra parte, se debe considerar que el alimento contenido en los microparches se presenta en forma discontinua en espacio y tiempo. Entonces, el encuentro con los microparches de abundancias discontinuas (bajo el umbral apropiado), se traduce en estados de condición intermedios (semisaludables), y en caso de fracasos sucesivos con sus presas se podría producir la inanición irreversible (Ivlev, 1961). Esta sería la explicación de los altos porcentajes de larvas en condiciones intermedias (semisaludables). Asimismo, las diferencias encontradas en las larvas capturadas a diferente distancia de la costa (estratos I, II, III, IV), confirmaría la idea que la oferta ambiental de alimentos es discontinua y heterogénea. En este estudio, la estructura física del océano mostró la condición de surgencia activa en el sector norte y de relajación en el sector sur (Fuenzalida, 1992). Estudios fitoplanctónicos bajo estas condiciones muestran que existe una composición diferencial de especies desde la costa (predominio de diatomeas) hacia afuera (dinoflagelados) (Herrera et al., 1992). Esta situación podría afectar la calidad del alimento para las larvas, lo cual podría explicar los diferentes estados de condición nutricional entre los estratos.

En resumen, el efecto de la condición nutricional es producto de la estructura ambiental que las larvas encuentran en determinado espacio y tiempo. Así, la condición fisiológica es el resultado de una historia de alimentación de éstas, donde la sobrevivencia dependerá de las adaptaciones particulares del individuo para capturar su alimento en ambientes tan dinámicos como el área de estudio. 
Entonces, para cualquier período de reclutamiento habrá muchos procesos físicos y biológicos, a diferentes escalas de espacio y tiempo, que influirían en el estado de condición de las larvas de Engraulis ringens.

Finalmente, el bajo porcentaje de larvas en condición de inanición, corroboraría la hipótesis que en un área de alta producción biológica, como ocurre frente a la desembocadura del río Loa, la inanición jugaría un rol secundario y habría que explorar otros procesos biológicos (depredación, canibalismo), como causas relevantes de mortalidad natural de larvas de esta especie.

\section{AGRADECIMIENTOS}

Los autores desean expresar su agradecimiento al Licenciado Sr. Guillermo Herrera por su ayuda en el análisis e interpretación de los estados nutricionales de las larvas, como asimismo, al Programa de Investigaciones Pesqueras Conjuntas (INPESCON, 1989), que permitió el financiamiento de este estudio.

\section{REFERENCIAS}

Blaxter, J.H.S. y G. Hempel. 1963. The influence of egg size on herring larvae. J. Cons. Perm. int. Explor. Mer., 28: 211-240.

Blaxter, J.H.S. y K.F. Ehrlich. 1974. Changes in behavior during starvation of herring and plaice larvae. In: J. Blaxter (ed): The Early Life History of Fish, Springer-Verlag, Berlin. pp. 277- 285.

Buckley, L. J. 1979. Relationships between RNADNA ration, prey density and growth rate in Atlantic cod (Gadus morhua) larvae. J. Fish. Res. Board Can., 36:1497-1502.

Buckley, L.J. 1984. RNA:DNA ration and index of larval fishgrowth in the sea. Mar. Biol., 80: 291298.

Clemmense, C.M. 1987. Laboratory studies en RNA/ DNA ration of starved and fed herring (Clupea harengus) y turbot (Scopthalmus maximus) larvae. J. Cons. Int. Explor. Mer., 43: 122-128.

Clemmense, C.M. 1988. A RNA and DNA fluorescence technique to evaluate the nutritional condition of individual marine fish larvae. Meeresforsch., 32: 134-143.
Ehrlich, K.F., H.S. Blaxter y R. Pemberton. 1976. Morphological and histological changes during the growth and starvation of herring and plaice larvae. Mar.Biol., 35: 105-118.

Frontier, S. 1966. Tratamiento de los datos. In: D. Boltovskoy (Ed): Atlas de zooplancton del Atlántico sudoccidental y métodos de trabajo con el zooplancton marino. INIDEP, Mar del Plata. pp. 169-188.

Fuenzalida, R. 1990. Variabilidad temporal de un índice de surgencia para la zona de Iquique (Lat. $20^{\circ} \mathrm{S}$ ). Invest. Cient. y Tec., Ser. Ciencias del Mar, 1: 37-47.

Fuenzalida, R. 1992. Proceso de surgencia en la región norte de Chile, latitudes $20^{\circ} 30^{\circ} \mathrm{S}-21^{\circ} 45^{\prime} \mathrm{S}$ ). Invest. Cient. y Tec., Ser. Ciencias del Mar, 2: 79104.

Govoni, J.J. 1980. Morpholological, histological and functional aspects of alimentary canal and associated organ development in larval Leiostomus xanthurus. Rev. Can. Biol., 39: 69-80.

Herrera, L. y C. Merino. 1992. Composición específica y abundancia relativa del fitoplancton marino del norte de Chile en 1989. Invest. Cient. y Tec., Ser. Ciencias del Mar, 2: 31-55.

Hjort, J. 1914. Fluctuations in the great fisheries of northern Europe viewed in the ligth of biological research. Rapp. P.V. Réun. Cons. Perm. int. Explor. Mer., 20: 1-228.

Hunter, J.R. 1972. Swimming and feeding behavior of larval anchovy, Engraulis mordax. Fish. Bull. U.S., 70: 821-838.

Ivlev, V.S. 1961. Experimental ecology of the feeding of fishes. Yale Univ. Press, New Haven, 302 pp.

Lasker, R. y J.R. Zweifel. 1978. Growth and survival of first-feeding northern anchovy larvae (Engraulis mordax) in patches containing different proportions of large and small prey. In: J.H. Steele (ed.). Spatial Pattern in Plankton Communities, Plenum Press, New York, pp. 329-354.

Martin, F.D. y R. Malloy. 1980. Histological and morphometric criteria for assessing nutritional state in larval striped bass Morone saxatalis. U.S. Fish and Wildlife Service Biological Services Program FWS/OBS-80, 43: 157-166. 
Martin, F.D. y D.A. Wright. 1987. Nutritional state and its use in pridicting stripe bass recruiment: Laboratory calibration. Amer. Fish. Soc. Symp., 2: $109-114$

O'Connell, C.P. y L.P. Raymond. 1970. The effect of food density on survival and growth of early post-yolk-sac larvae of the northern anchovy (Engraulis mordax Girard) in the laboratory. J. Exp. Mar. Biol. Ecol., 5: 187-197.

O'Connell, C.P. 1976. Histological criteria for diagnosing the starving condition in early post yolk-sac larvae of the northern anchovy, Engraulis mordax Girard. J. Exp. Mar. Biol. Ecol., 25: 285-312.

O'Connell, C.P. 1981. Estimation by histological methods of the percent of starving of the northern anchovy (Engraulis mordax) in the sea. Rapp. P.V. Réun. Cons. int. Explor. Mer., 178: 357-360.

Owen, R.W. 1980. Patterning of flow and organisms in the larval anchovy environment. In:Workshop on the effects of environmental variation on the survival of larval pelagic fishes. UNESCO, FAO, IOC Workshop Rep., 28: 167-200.

Owen, R.W. 1981a. Microscale plankton patchiness in the larval anchovy environment. In: R. Lashker and K. Sherman (eds.). The Early Life History of Fish., Rapp. P.V. Réun. Cons. int. Explor. Mer., 178: 364-368.

Owen, R.W. 1981b. Microscale patchinness of small plankton on the Chimbote Shelf, Perú. Bol. Inst. Mar. Perú-Callao (IMARPE), Vol. extraordinario: 274-279.

Palma, W., J. Pizarro y C. Flores. 1992. Co-ocurrencia, distribución y abundancia de los estados tempranos de Engraulis ringens y Sardinops sagax (Pisces:Clupeiformes) en un área de surgencia costera en el norte de Chile. Invest. Cient. y Tec., Ser. Ciencias del Mar, 2: 12-30.
Santander, H. y R. Flores. 1983. Los desoves y distribución larval de cuatro especies pelágicas y sus relaciones con las variaciones del ambiente marino frente al Perú. FAO. Fish. Rep., 291(3): 835-868.

Smith, P.E y S.L. Richardson. 1979. Técnicas estándar para prospecciones de huevos y larvas de peces pelágicos. FAO, Doc, Téc. Pesca, 175: 1107

Sokal, R y J. Rohlf. 1969. Biometry. W.H. Freman. Co. San Francisco, C.A., 776 pp.

Theilacker, G.H. 1978. Effect of starvation on the histological and morphological characteristics of jack mackerel, Trachurus symmetricus, larvae. Fish. Bull., 76: 403-414.

Theilacker, G.H. 1986. Starvation-induced mortality of young sea-caught jack mackerel, Trachurus symmetricus, determined with histological and morphological methods. Fish. Bull., 84: 1-17.

Theilacker, G.H. y Y. Watanabe. 1989. Midgut cell heigth defines nutritional status of laboratoryraised larval northern anchovy, Engraulis mordax. Fish. Bull., U.S. 87: 1989-1998.

Umeda, S. y A. Ochiai. 1975. On the histological structure and function of digestive organs of the fed and starved larvae of the fed and starved larvae of the yellowtail, Seriola quinqueradiata Jap. J. Ichthyol., 21: 213-219.

Uriarte, I. y F. Balbontín. 1987. Caracterización del estado de hambruna en las larvas de sardina Sardinops sagax musica (Pisces, Clupeiformes) mediante criterios morfométricos e histológicos. Rev. Biol. Mar. Valparaíso, 23(1): 77-106.

Wright, D.A. y F.D. Martin. 1985. The effect of starvation on RNA:DNA rations and growth of larval striped bass, Morone saxatilis. J. Fish. Biol., 27: 479-485. 